\title{
Data System of the Finnish Profitability Bookkeeping
}

\author{
Sami Chaudhary ${ }^{1)}$ and Olli Rantala ${ }^{1)}$ \\ ${ }^{1)}$ MTT Economic Research, Latokartanonkaari 9, 00790 Helsinki, first name.last name@ mtt.fi
}

\begin{abstract}
MTT Economic research monitors the economy and financial development of agricultural and horticultural enterprises and reindeer farms on the basis of farm level accountancy data. Around 1000 enterprises from all over Finland are voluntarily participating the profitability bookkeeping providing their bookkeeping data to be used as research and statistical material.

During the long history since 1912 and especially in recent decades the data system has gone through many technical changes from manual systems to desktop adb-systems and finally to internet based web application. The system development work has also produced many significant innovations.

The data of farms has been recorded since 1998 by using dedicated bookkeeping program martti, which is a windows based desktop application. We are now in the process of deploying the existing application to a web application so that the data can be stored online over internet. The recent PowerBuilder versions allow deploying the existing PowerBuilder desktop applications as web applications after making required changes in the application code. PowerBuilder uses the .NET infrastructure to generate the ASP.NET web application.

By taking advantage of this feature of PowerBuilder, we have deployed our desktop application martti to the web application. The web application consists of three tier architecture: the front end with the client browser, PowerBuilder components on the Internet Information Services (IIS) server and the back end database to store the data.

Web applications have several advantages over traditional client-server applications relating to i.e. installation, application maintenance, dynamic settings and management.

The simple PowerBuilder applications work on the internet after deploying without problems, however the complex applications may not work smoothly on the internet. Because of complex architecture of martti application, there are also some challenges and limitations to deploy and use as a web application.
\end{abstract}

Key words: Profitability bookkeeping, data system, datawarehouse 


\section{Introduction}

MTT Economic research monitors the economy and financial development of agricultural and horticultural enterprises and reindeer farms on the basis of farm level accountancy data. Around 1000 enterprises from all over Finland are voluntarily participating the profitability bookkeeping providing their bookkeeping data to be used as research and statistical material.

MTT Economic research is responsible for collecting the data and maintaining and developing the information systems to process and store the data and to disseminate the results. The Business Accountancy team conducts the profitability research and produces various average and simulation results as well as estimates. The Accountancy team also develops profit calculation procedures for the diversified and changing agriculture: calculation frameworks and financial indicators depicting the operational requirements of the sector. The material and results are widely utilised by research, administration, advising, teaching and farmers.

The Finnish profitability bookkeeping is part the EU's statutory farm accountancy data network (FADN). MTT acts as the national liaison agency of FADN and provides the Commission annually with the Finnish set of farm level data. The FADN system is a key instrument in planning and monitoring the common agricultural policy (CAP) of the EU.

During the long history since 1912 and especially in recent decades the data system has gone through many technical changes from manual systems to desktop adb-systems and finally to internet based web application. The system development work has produced many significant innovations.

The results from 1998 on have been published in MTT's EconomyDoctor Internet website which consists of several web services.

\section{Data storage with Martti desktop application}

The data of farms has been recorded since 1998 by using the bookkeeping program martti, which is a windows based desktop application. The data recorded consist of proper bookkeeping data such as cash receipts and expenditures, but also quite detailed data of fixed and current assets, personnel and labour input, use of cultivated land, animals and crops and yields. The financial statement is prepared on so called accrual basis, where the returns and costs are matched to the same year i.e. the year when the output is produced.

The farm level data from martti program is then checked and processed and transferred to production database (DW) using dedicated applications, made with the PowerBuilder.

We are now in the process of deploying our existing application to a web application so that the data can be stored online over internet. The following paragraphs describe some of the technical details and overview of the new online system.

The martti application was developed with the PowerBuilder, an integrated development environment by Sybase which allows developing user interfaces and application processing logic of client-server applications using the PowerScript language.

\section{Online data storage with Martti application}

The recent PowerBuilder versions allow deploying the existing PowerBuilder desktop applications as web applications after making required changes in the application code. PowerBuilder uses the .NET infrastructure to generate the ASP.NET web application.

By taking advantage of this feature of PowerBuilder, we have deployed our desktop application martti to the web application. The web application consists of three tier architecture: the front end with the client browser, PowerBuilder components on the Internet Information Services (IIS) server and the backend database to store the data. 


\section{Advantages of the online application}

Web applications have several advantages over traditional client-server applications. Following are few of the advantages which martti web application offers.

\section{No installation}

The desktop application needs to be installed on each of the computer on client side while the web application does not need to be downloaded or installed on client-side. It can be accessed easily with a URL using the web browser.

\section{Application maintenance}

As the desktop application needs to be installed separately on each computer, therefore updating the application is very complicated, which requires un-installation and re-installation of the application on each of the computer. However, the web application is very easy to maintain, as it needs to be installed only on the web server.

\section{Dynamic settings}

The desktop application stores the user settings only in the local computer, where the application is installed. However in the martti web application, the user preferences are stored in the database on the web server, so that wherever user accesses the application, the application retrieves the settings.

\section{Centralized database}

The martti desktop application uses a small database file on each of the computer where the application is installed, while the web application uses a centralized database located on the web server.

\section{Challenges in the online application}

The simple PowerBuilder applications work on the internet after deploying without problems, however the complex applications may not work smoothly on the internet. Because of complex architecture of martti application, there are some challenges and limitations to deploy and use as a web application.

\section{Software limitations}

PowerBuilder supports most of the functions in the web applications, such as basic DataWindow and embedded SQL. However still some functions such as data pipeline and drag-and-drop are not supported.

When an application is deployed to web application, PowerBuilder reports all unsupported functions which have been used in the desktop application. Those functions need to be modified in order to make application work on the internet.

\section{Browser dependency}

The PowerBuilder deployed web applications are supported to function only within the Internet Explorer. The interface does not display properly on other browsers. 


\section{Data transfer}

The martti application uses the PowerBuilder data pipeline function transfer data from one database to another database. The data pipeline function gives ability to reproduce the data within the database or across databases. However, this function is supported only in the desktop application. Therefore the data transfer from one database to another is not possible using the web application.

\section{State management}

The web application is accessed with the HTTP, which is a stateless protocol. Which means, when the user enters the information in the form fields and send the form to the server, if the form is not successfully submitted and displayed again because of some error, the information filled in the form will be lost.

\section{Internet connectivity and bandwidth}

The web application relies significantly on the internet connectivity and bandwidth. The bandwidth refers to the amount of the data which can be transmitted in a specific time. Absence of internet connection or its poor bandwidth can cause performance issues and the lost of data.

\section{Privacy and Security}

As the web application uses a centralized database located on the web server, it is important to maintain the users' privacy and database security. For example, the users should be able to access their own data only. Also, internet applications are exposed to more security threats than the stand alone desktop applications.

\section{System overview}

The web application is hosted on the dedicated windows based web server and is connected to the database located on the database server. Accounting officers can access the web application with the URL. After successful login, they can access to their farm data, and fill the data.

The application has a built-in feature to validate the data, which works by connecting to the checking server via FTP server, where several tests are done using a separate testing application. The validation process works same as in desktop application.

Once the data is submitted after corrections, it is stored initially in the database server. The researchers can connect to the web server, and download the data to their local computers for checking, the data is finally stored to the production database server.

The following diagram provides an overview of the web application system: 


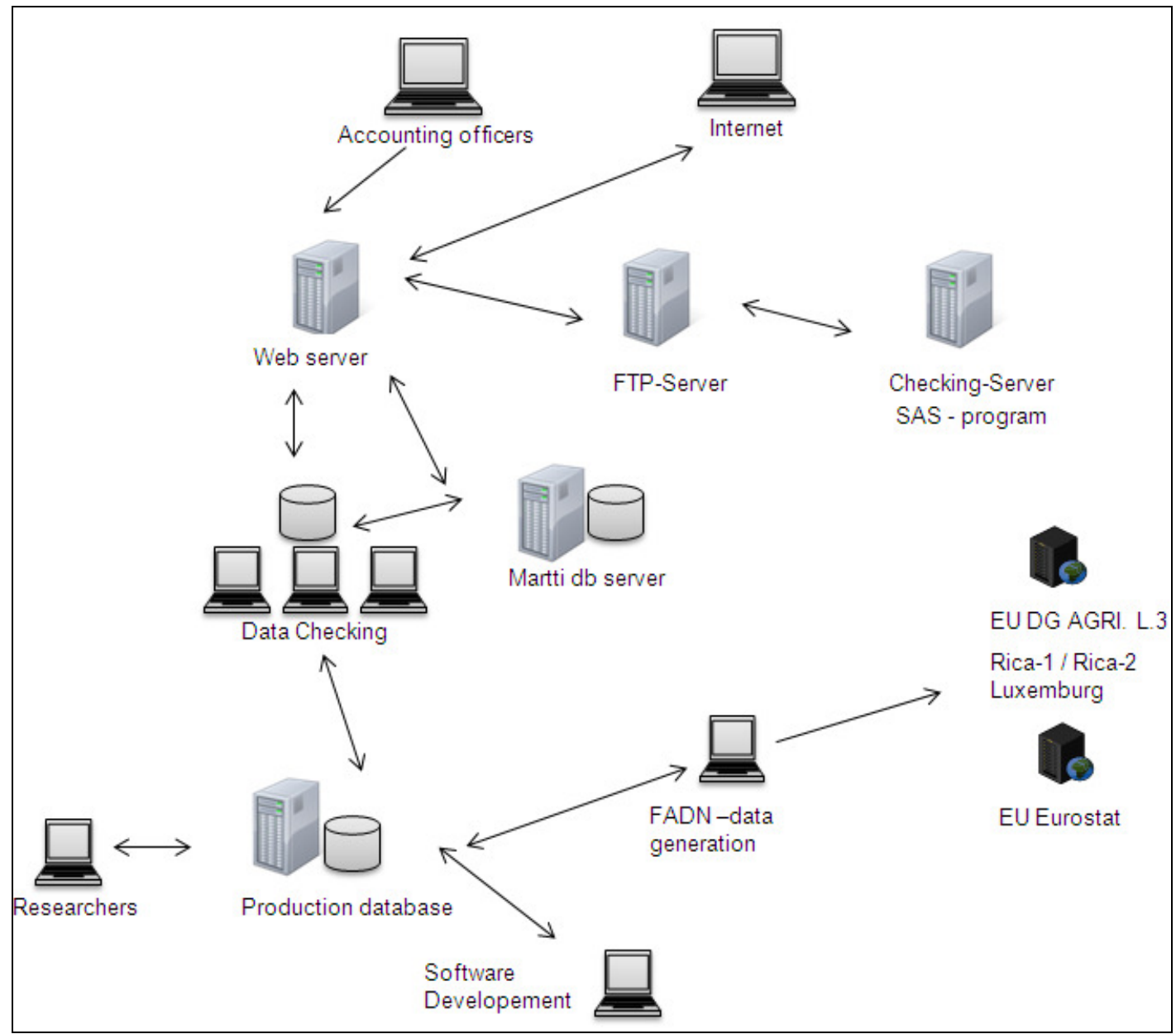

Fig 1. An overview of the web application system used as data system in Finnish profitability bookkeeping. 